\title{
Physiological characterization and thylakoid ultrastructure analysis in super high-yield hybrid rice leaves under drought stress
}

\author{
Y.W. WANG ${ }^{\dagger}$, D.X. JIANG ${ }^{\dagger}$, J.J. HOU, and G.X. CHEN ${ }^{+}$ \\ Jiangsu Key Laboratory of Biodiversity and Biotechnology, Life Sciences College, Nanjing Normal University, \\ 210023 Nanjing, China
}

\begin{abstract}
Rice serves for more than a third of the world's population. However, drought stress (DS) affects its growth and yield. In order to understand plant drought adaptability, we explored the physiological and thylakoid ultrastructural alteration in super high-yield hybrid rice Liangyoupeijiu (LYPJ) under DS. Results showed that DS altered the thylakoid ultrastructure; twisted and fractured stroma thylakoids and more starch granules in chloroplast of LYPJ were present. Also, DS lowered chlorophyll and carotenoid contents in LYPJ after $7 \mathrm{~d}$. DS reduced the proportion of unsaturated fatty acids in thylakoid membranes and increased the content of linoleic and abscisic acids (ABA) and osmotic substances such as calcium and potassium in LYPJ. Based on these results, we concluded that although DS severely affected normal growth, plants employed various strategies, such as changing their membrane lipid fluidity, increasing endogenous ABA and osmotic substance contents and stabilizing the grana thylakoids, in order to adapt to drought stress.
\end{abstract}

Additional key words: abiotic stress; membrane lipid fluidity; osmotic adjustment; phytohormone.

\section{Introduction}

Different varieties of hybrid rice are widely planted for their high yield potential (Cheng et al. 2007). Liangyoupeijiu (LYPJ) is one of the typical representatives of high-yield hybrid rice in China. Nonetheless, similar to other hybrid rice cultivars, premature senescence in LYPJ caused by intrinsic genetic factors, accelerated by adverse environmental stress, results in a decline in photosynthesis during the late growth which may have devastating effects on the stability of high yield, especially, at the flowering and milk stage (Zhang et al. 2010). It must be also mentioned that LYPJ as a mid-late maturing variety shows slower grain-filling rate compared to other intrasubspecific hybrids (Yang and Zhang 2006). This means that the potential risk of water deficit may occur since the flowering to grain-filling stage at any time.

Drought is considered as one of the most damaging climate-related hazards due to the phenomenon of global warming, and has been a direct impediment for crop cultivation areas and yield (Lanna et al. 2018). Under drought stress, the loss of water and the accumulation of peroxide lead to serious damage to chloroplasts. Degradation of chloroplasts in plants resulted in net photosynthetic rate decline and growth retardation (Varone et al. 2012). On the other hand, plants can employ a series of physiological and biochemical mechanisms which contribute to drought resistance. Changes in the content and composition of fatty acids, important components of membranes, in order to alleviate the damage caused by drought stress, have been intensively investigated (Gondor et al. 2014). However, little information is known on the fatty acids alteration in the thylakoid membranes. As a phytohormone, abscisic acid (ABA) can accumulate rapidly in response to stress, regulate a variety of stress responses, and adapt plants to stress (Ullah et al. 2018). In recent years, various signal factors, such as cytoplasmic calcium $\left(\mathrm{Ca}^{2+}\right)$, phospholipase $\mathrm{C}$ (PLC), and other signaling molecules (Li et al. 2017) involved in ABA signaling, have been identified and studied at individual, tissue, and cellular levels in plants under environmental stress. In addition, osmotic adjustment (OA) is thought to be related to drought tolerance and found to be useful in other species such as wheat (Abid et al. 2018). The major consequence of OA is the maintenance of turgor potential at low leaf water potential. During drought stress, some osmotic adjustment substances including $\mathrm{Ca}$, $\mathrm{K}, \mathrm{Na}$, and $\mathrm{S}$ significantly accumulate, thereby regulating

Received 14 March 2019, accepted 26 June 2019.

${ }^{+}$Corresponding author; phone: +86-25-85891236, e-mail: gxchennjnu@hotmail.com

Abbreviations: ABA - abscisic acid; Car - carotenoids; CAT - catalase; Chl - chlorophyll; DS - drought stress; FM - fresh mass; HPLC - high-performance liquid chromatography; IAA - indole-3-acetic acid; IUFA - index of unsaturated fatty acid; OA - osmotic adjustment; PLC - phospholipase C; ROS - reactive oxygen species; RWC - relative water content; SOD - superoxide dismutase; TEM - transmission electron microscope; WW - well-watered.

Acknowledgements: This work was supported by the Natural Science Foundation of Jiangsu province of China (BK20171034), the Agricultural Independent Innovation Foundation of Jiangsu Province (CX173022), and the Priority Academic Program Development of Jiangsu Higher Education Institutions (PAPD).

These authors contributed equally to this work. 
the osmotic potential of grapevine leaves (Patakas et al. 2002). However, further research is needed to elucidate how these factors contribute to water-stress tolerance in some widely cultivated high-yield hybrid rice cultivars during the grain-filling period which coincides with the occurrence of the flag leaf senescence.

Many studies on the reactive oxygen species (ROS) and antioxidant enzymes in plants under drought stress have been reported (Khanna-Chopra 2012). However, there is little systematic study on ABA, osmotic adjustment substances, and fatty acid content combined with thylakoid ultrastructure analysis at the flag leaf senescence stage in rice. Furthermore, most studies on drought have been performed with rice species grown under artificial or simulated conditions. We hypothesized that rice LYPJ could confer an advantage in heterosis of hybrid rice in the senescence stage of flag leaf by increasing osmotic adjustment substances, changing membrane structure and fatty acid composition as well as endogenous hormone contents. In order to test this hypothesis, we carried out a study of the physiological and thylakoid ultrastructural alterations under natural drought conditions instead of PEG 6000-induced drought stress. Our aim was a better understanding of leaf senescence processes and adaptation and adjustment mechanism in the modern high yield rice cultivar LYPJ under drought stress.

\section{Materials and methods}

Plant materials and growth conditions: The experiments were conducted from 10 August (first flag leaves expansion) to 26 September (the harvest time) in 2014 and 2015 in Nanjing Normal University, Jiangsu Province, China. Seedlings of LYPJ with equal growth (the third leaf fully expanded) were procured from the nursery of botanical garden. Potted seedlings were arranged in a single-factor randomized block design, with three replications of each treatment when the first flag leaves expanded. Two treatments were applied: well-watered (WW) and droughtstressed (DS). Pot water holding capacity was maintained at $100 \%$ for WW but withheld in DS. DS treatment was initiated on rice with the flag leaves fully expanded by withholding water; based on leaf curling symptoms. All plant were well watered before DS treatment. The soil relative water content (RWC) was maintained less than $35 \%$ after irrigation for DS plants to ensure stress status. During the experimental treatment period, samples were collected at about $7-\mathrm{d}$ intervals and stored at $-80^{\circ} \mathrm{C}$ until analysis.

Chlorophyll (Chl) and carotenoid (Car) contents were extracted from $0.5 \mathrm{~g}$ leaf samples using $80 \%$ acetone; absorbances at 470,645, and $663 \mathrm{~nm}$ were recorded on a spectrophotometer (Genesys 10, Thermo Electron, USA). The calculations of $\mathrm{Chl}$ and Car contents were done according to the method described by Arnon (1949) and Lichtenthaler (1987).

Chloroplast ultrastructure: The flag leaves without the leaf midrib were cut into small pieces (approximately
$0.1 \times 0.5 \mathrm{~cm}$ ). These small pieces were placed in a bottle with $4 \%$ glutaraldehyde buffer solution, and the air was pumped out of the bottle with a syringe so that the leaves became fully soaked in the buffer solution. Leaves were fixed at $4^{\circ} \mathrm{C}$ for over $24 \mathrm{~h}$. They were then rinsed in phosphate buffer ( $\mathrm{pH}$ 7.4) for $15 \mathrm{~min}$ and post-fixed in $5 \%$ osmium tetroxide at the room temperature. The fixed samples were dehydrated in an ascending series of acetone $(30,50,70,80$, and $90 \% ; 15$ min each $)$ and in $100 \%$ acetone (three times by 7-8 $\mathrm{min}$ ), washed in $100 \%$ acetone for $15 \mathrm{~min}$, and embedded in the Epon 812 resin. Thin sections were obtained with an $L K B-V$ ultramicrotome ( $L K B$ Ultrascan XL, Bromma, Sweden) and double stained with uranyl acetate-lead citrate before being examined with a transmission electron microscope (TEM) (Hitachi 600- $A-2$, Japan) operating at $75 \mathrm{kV}$.

Thylakoid membrane lipids were analyzed according to the method of Zhang et al. (2010). The extract of chloroplast membrane lipids was surged several times with $1 \mathrm{~cm}^{3}$ of $0.4 \mathrm{M}$ methanol solution and kept motionless for $2 \mathrm{~h}$. The mixture was extracted with $10 \%$ acetic acid and heptane. The upper solution was imbibed and evaporated to dryness under reduced pressure. The extract, with the addition of some chloroform, was then quantitatively analyzed by gas chromatography (G1530N, Agilent, USA) A chromatographic data processor was used to record and store chromatograms and to calculate peak areas. Peaks were identified through comparison with several external qualitative standards.

Inorganic element contents in the leaf cells and their different cell components including cell wall, organelles, and cytoplasm were determined by flame atomic absorption spectrometry (SpectrAA220FS, USA). The components of cells were separated according to the method by Shi and Pan (2015). All the samples were dried well and put into digestive tubes. Each digestive tube was immersed in perchloric acid $(5 \mathrm{ml})$ and concentrated nitric acid $(15 \mathrm{ml})$ overnight. Every tube was digested at $180^{\circ} \mathrm{C}$ until no impurities, and then water was added to obtain a volume of $50 \mathrm{ml}$.

Determination of ABA and IAA: The measurement of ABA was conducted according to the method described by Zhou et al. (2016) with some modifications. In brief, $2 \mathrm{~g}$ of leaf tissues were collected, ground in liquid nitrogen, extracted in $80 \%$ methanol $(\mathrm{v} / \mathrm{v})$, purified, dissolved in $600 \mu \mathrm{l}$ of high-performance liquid chromatography (HPLC) mobile phase (acetonitrile:acetic acid (1.8\%), $1: 1, \mathrm{v} / \mathrm{v})$, and filtered $(0.22 \mu \mathrm{m})$ for endogenous ABA determination. The ABA concentration was analyzed using an Agilent 6540 Accurate Mass LC-MS Q-TOF. The indole-3-acetic acid (IAA) concentration in rice leaves was determined using an LC/MS method after extraction, purification, and filtration $(0.22 \mu \mathrm{m})$ as described by Vayssières et al. (2015).

Statistical analysis: All data were statistically analyzed with SPSS (version 18.0, USA). The data shown are mean 
value \pm standard deviation (SD). Differences between treatment means were compared using the LSD at the 0.05 probability level. Single and double asterisks refer to significant difference between the drought and the control at 0.05 and 0.01 , respectively.

\section{Results}

Chl and Car contents: As shown in Fig. 1, when exposed to drought treatment for $7 \mathrm{~d}, \mathrm{Chl}$ and Car contents were slightly higher than that of WW plants. From 14 d, both Chl and Car contents decreased significantly compared with the WW group. After $35 \mathrm{~d}$ of drought stress, the minimum $\mathrm{Chl}$ and Car contents were observed in DS group.

Chloroplast and thylakoid ultrastructure: Changes in chloroplast ultrastructure were observed in WW and DS plants. At $14 \mathrm{~d}$, mesophyll cells in flag leaves of WW plants possessed considerable plump chloroplasts, which contained grana with appressed stacking and welldeveloped stroma lamellae (Fig. 2A,B). Following DS group, a significant increase of starch granules in chloroplast was noticed and plastoglobules showed a high electron density (Fig. 2E,F). In DS plants, chloroplasts changed their shape to a smaller oval, with typical fine structure of grana stacks shrank in plies and curved or partially cracked stroma lamellae. Additionally, the cell walls of DS plants showed remarkable thickenings. Up to $28 \mathrm{~d}$, WW plants exhibited loosely appressed and deformed grana, along with large plastoglobules (Fig. 2C,D). Interestingly, DS plants presented smaller but appressed grana stacks with swollen lumen and chloroplasts no longer clung to the cell membrane. In addition, stromal thylakoids twisted and fractured, while granal thylakoids still kept compact (Fig. 2G,H). Furthermore, we found several concentric inner membrane structures with crystalline inclusions in the cytoplasm of DS plants (Fig. $2 G$ ).

The fatty acids of thylakoid membrane lipids were separated and analyzed by gas chromatography. As shown in Fig. 3, two saturated fatty acids, palmitic acid (c16:0) and stearic acid (c18:0), and four unsaturated ones,

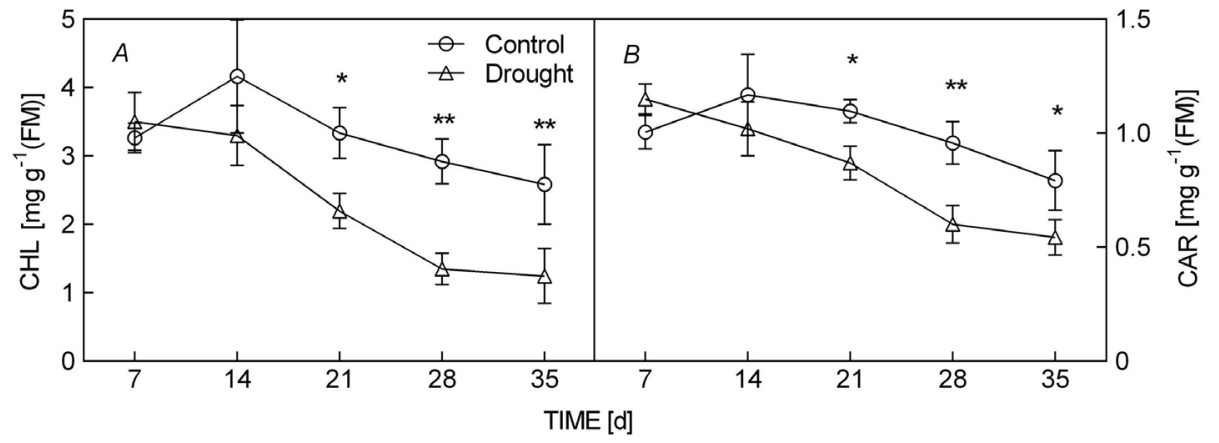

Fig. 1. Changes in chlorophyll $(\mathrm{Chl})(A)$ and carotenoid (Car) $(B)$ contents of flag leaves during senescence stage in high-yield hybrid rice LYPJ exposed to drought stress for $35 \mathrm{~d}$. Vertical bars represent SD $(n=3)$. Single and double asterisks refer to significant difference between the drought and the control at 0.05 and 0.01 , respectively. FM - fresh mass.
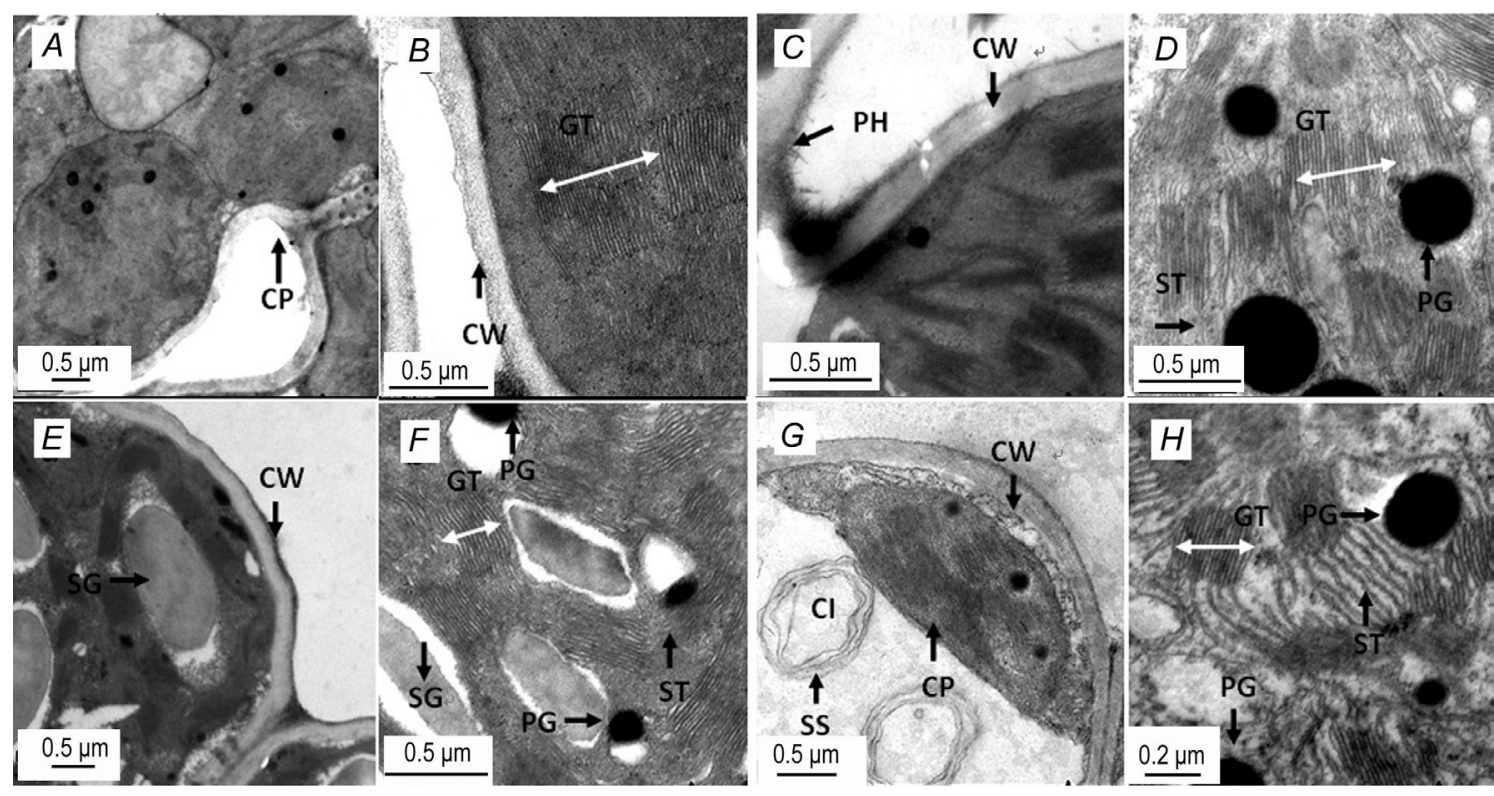

Fig. 2. Transmission electron micrographs of chloroplasts and thylakoids in leaf mesophyll cells of rice in control and drought-stressed plants. Control $(A, B)$ and drought-stressed $(E, F)$ plants at low $(A, E)$ and high $(B, F)$ magnification on $14 \mathrm{~d}$. Control $(C, D)$ and droughtstressed $(G, H)$ plants at low $(C, G)$ and high $(D, H)$ magnification on $28 \mathrm{~d}$. CI - crystalline inclusions, $\mathrm{CP}$ - chloroplast, $\mathrm{CW}$ - cell wall, GT - grana thylakoid, PG - plastoglobuli, PH - phenols, SG - starch granule, SS - special structure, ST - stroma thylakoid. 
palmitoleic acid (c16:1), oleic acid (c18:1), linoleic acid (c18:2), and linolenic acid (c18:3) among the thylakoid membrane lipids were found. Different patterns of fatty acid composition of thylakoid membranes were found. When exposed to DS treatment for $14 \mathrm{~d}$, compared with that of the control plants, the stearic acid content of the thylakoid membranes increased, but oleic acid was significantly reduced by $39.6 \%$ (Fig. $3 A$ ). While on $28 \mathrm{~d}$, palmitic acid content increased and linoleic acid showed an increment up to $35.1 \%$ (Fig. 3B). Moreover, DS significantly decreased the linolenic acid content by 8.5 and $17.4 \%$ at the two time points, respectively. Specifically, the ratio of unsaturated to saturated fatty acids, index of unsaturated fatty acid (IUFA), was reduced by DS treatment compared with control plants (Fig. 3C).

The inorganic element concentrations in leaf cell, cell wall, organelles, and cytoplasm of two groups were measured by flame atomic absorption spectrometer at $21 \mathrm{~d}$ (Fig. 4). Compared with WW plants, the concentrations of $\mathrm{K}, \mathrm{Ca}$, and Mo in cell walls of DS plants significantly increased by $20.9,49.7$, and $83.0 \%$, respectively (Fig. $4 A$ ). As shown in Fig. $4 B$, the concentration of $\mathrm{Mg}$ in DS organelles significantly decreased by $56.3 \%$ and $\mathrm{Ca}$ increased by $19.6 \%$. The concentrations of $\mathrm{P}, \mathrm{K}$, and $\mathrm{Ca}$ in DS cytoplasm increased by $35.5,21.2$, and $84.8 \%$, respectively, while $\mathrm{S}$ decreased by $20.3 \%$ (Fig. $4 C$ ). As shown in Fig. $4 D$, the changes of inorganic ion concentrations in DS leaf entire cells were similar to that in cytoplasm. The concentrations of $\mathrm{P}, \mathrm{K}$, and $\mathrm{Ca}$ increased by $182.5,25.2$, and $49.7 \%$, while $\mathrm{Mg}$ and Mo decreased by 34.7 and $20.3 \%$, respectively.

ABA and IAA content: Under DS treatment, ABA content of leaves increased significantly and was higher than that in the control during the whole experimental period (Fig. 5). At 7, 21, and $35 \mathrm{~d}, \mathrm{ABA}$ content of leaves increased by $139.3,112.9$, and $225.5 \%$ under DS compared with control (Fig. $5 A$ ). However, there was no difference in IAA content between the WW and DS plants (Fig. $5 B$ ).

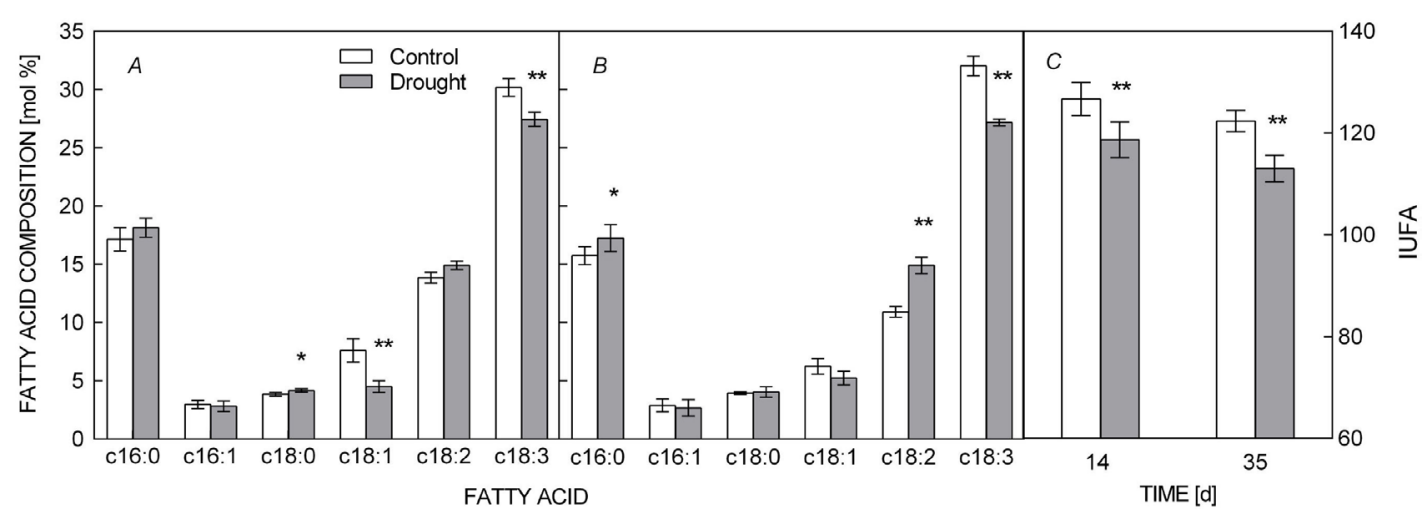

Fig. 3. Fatty acid composition analysis conducted on plants on $14(A)$ and $28 \mathrm{~d}(B)$, index of unsaturated fatty acid $(C)$ after full expansion of flag leaves in high-yield hybrid rice LYPJ under drought treatment. Vertical bars represent SD $(n=3)$. Single and double asterisks refer to significant difference between the drought and the control at 0.05 and 0.01 , respectively.

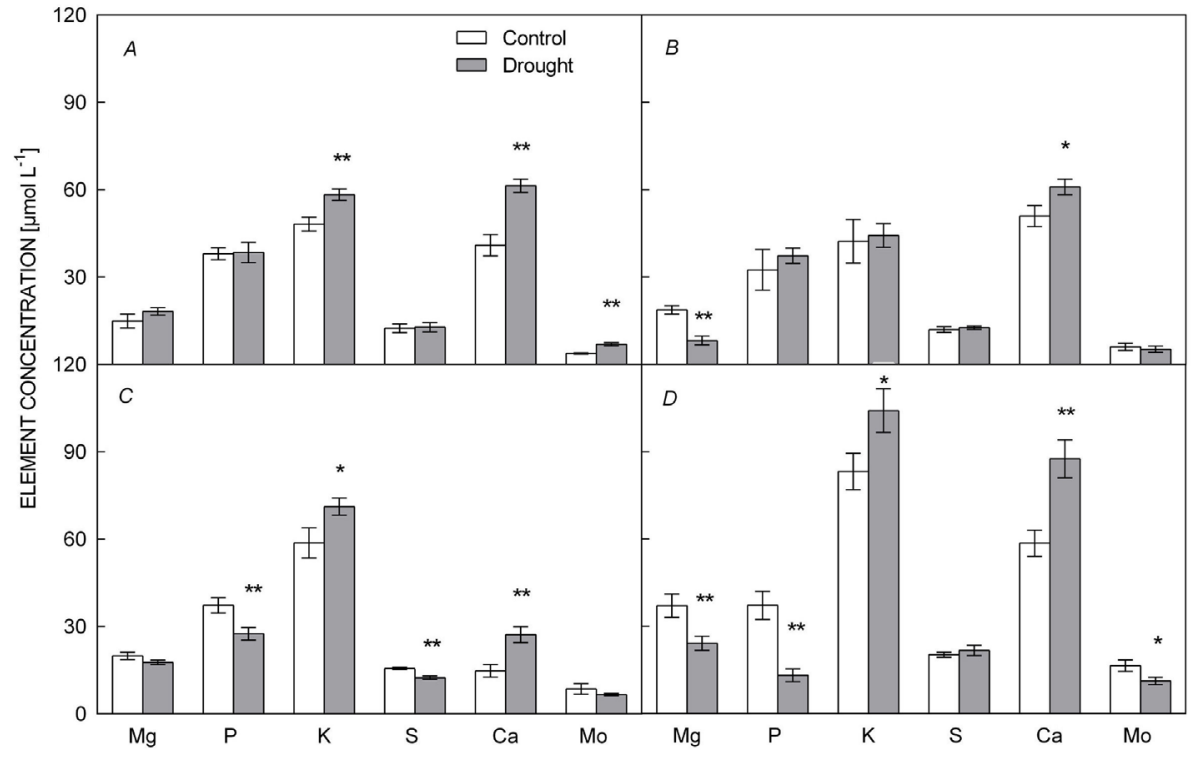

Fig. 4. Changes in inorganic element concentration in the cell wall $(A)$, organelles $(B)$, cytoplasm $(C)$, and entire cell $(D)$ of flag leaves during senescence stage in high-yield hybrid rice LYPJ exposed to drought stress. Vertical bars represent SD $(n=3)$. Single and double asterisks refer to significant difference between the drought and the control at 0.05 and 0.01 , respectively. 


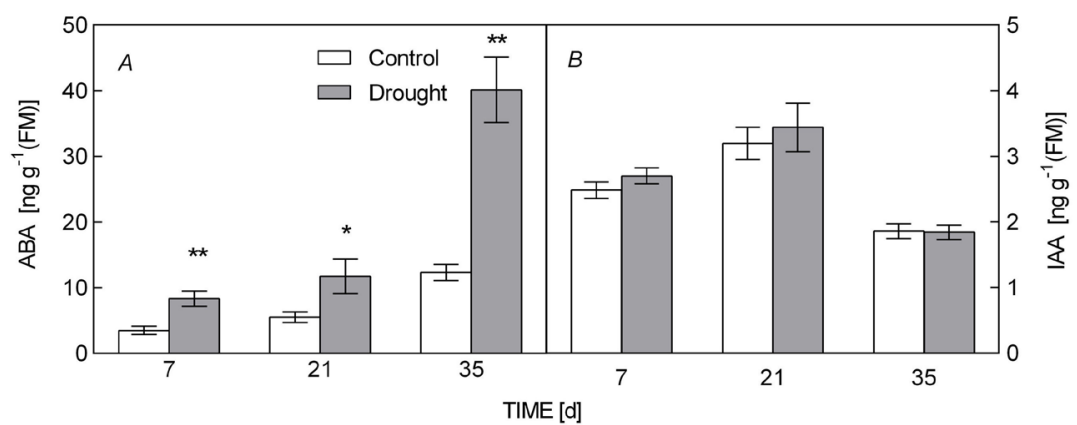

Fig. 5. Changes in abscisic acid (ABA) $(A)$ and indole-3-acetic acid (IAA) $(B)$ of flag leaves during the senescence stage in high-yield hybrid rice LYPJ exposed to drought stress. Vertical bars represent SD $(n=3)$. Single and double asterisks refer to significant difference between the drought and the control at 0.05 and 0.01 , respectively. FM - fresh mass.

\section{Discussion}

Chl decomposition is the initial process of leaf senescence, while DS accelerates leaf senescence and the loss of photosynthesis (Avila-Ospina et al. 2014). LYPJ had a rapid stress response and the $\mathrm{Chl}$ content increased in the early stage of DS. However, as the period of DS was extended, the contents of leaf $\mathrm{Chl}$ and Car decreased significantly (Fig. 1). A decrease in Chl content might bring about photooxidation and degradation of photosynthetic apparatus, which is a representative phenomenon of oxidative stress (Farooq et al. 2009). Moreover, it is already known that Car act as efficient quenchers of trianions of $\mathrm{Chl}$ and singlet oxygen and are also precursors of the ABA. The decreased Car in LYPJ rice under DS in our study indicated that the plant was easily prone to light damage (Cunningham and Gantt 1998, Sultana et al. 1999). In addition, the Car content in DS plants was higher than that of the control group during the early stages of DS in our work. We considered that this fact probably represented an increase of Car biosynthesis in the DStreated rice during the early stages of drought stress, which would be necessary to compensate a possible $\mathrm{Chl}$ reduction induced by a longer exposure to drought conditions.

Although our previous studies investigated the ultrastructure of chloroplasts in hybrid LYPJ during the grainfilling period (Chen et al. 2004), we focused more on the thylakoid ultrastructure in this study at the flowering and milk stage under DS. At $14 \mathrm{~d}$, starch granules increased significantly and a small number of osmiophilic granules formed in chloroplasts of DS group. Chloroplasts under DS changed their shapes to a smaller oval and grana stacks with swollen lumen and were no longer clung to the cell membrane. Furthermore, thylakoids in DS at $28 \mathrm{~d}$ were swollen and there was a disarrangement of the stromal lamellae (Fig. 2). These morphological changes were similar to those observed by Yamane et al. (2003). It should also be noted that DS induced a lot of starch granules accumulating in chloroplasts (Fig. $2 F$ ), suggesting that the pathway of transport of the energy, which was fixed by leaf photosynthesis, to the seed was destroyed. Due to the severe loss of water during the drought, the stroma thylakoids of DS were loosely bent in the chloroplast (Fig. 2H), which was in accordance with Shao et al. (2016). Interestingly, stromal thylakoids twisted and broke, but the granal thylakoid lamellae remained relatively tight (Fig. $2 H$ ). This indicated that the structure and function of LHCII still kept intact and the DS-induced photoinhibition was eliminated by dissipation of excitation energy as heat (Hu et al. 2013).

There is a general consensus that the fatty acids are essential components for different cellular membranes that provide structural barriers to the environmental stress (Upchurch 2008). The proportion of saturated fatty acids in thylakoid membranes increased under DS (Fig. $3 A, B$ ), which was identical with previous study of Gondor et al. (2014). Thomas et al. (1986) suggested that mature leaves increase the melting temperature of the plasma membrane by elevating saturated fatty acids to enhance the heat resistance of plants. In addition, the IUFA of DS decreased sharply by DS treatment compared with WW plants at both 7 and $28 \mathrm{~d}$ (Fig. 3C). Wang et al. (2010) reported that a smaller reduction in IUFA under stress was propitious to the stability of the thylakoid membrane. However, the linoleic acid (c18:2) in DS increased significantly at $28 \mathrm{~d}$ (Fig. 3B), which was similar to our previous study, where a large amount of linoleic acid accumulated in the senescent leaves of LYPJ (Zhang et al. 2010). We speculated that the increase of linoleic acid can aggrandize the fluidity of the thylakoid membranes to improve the repair rate of PSII in crops under severe drought stress.

It is well known that many plants have reduced their osmotic potential in order to adapt to drought conditions (Patakas et al. 2002). The accumulation of elements during drought stress is really interesting. The concentration of Ca increased significantly in stressed plants (Fig. 4). The rapid increase in $\mathrm{Ca}$ concentration can affect tissue elasticity and increase the rigidity of the cell wall (Atkinson 1991). This was in agreement with the considerable increase in thickness of cell walls observed in LYPJ under water stress (Fig. 4). Ca also plays a key role in stabilizing plant membranes and cell walls by attaching various proteins and lipids to the membrane surfaces (Hirschi 2004).

Osmotic adjustment is associated with plant drought tolerance ability (Babu et al. 2008, Guo et al. 2018). Potassium exists mainly in the form of ions. The $\mathrm{K}^{+}$ concentration increased significantly in DS because $\mathrm{K}^{+}$is well-known as osmoticum (Fig. 4). The result indicated that the increasing $\mathrm{K}^{+}$concentration cloud employs both functions of decreasing the osmotic potential and enhancing stomatal conductance (Blatt and Gradmann 1997). However, with deficiency in soil moisture, $\mathrm{P}$ concentration decreased significantly in stressed plants. When the concentration of $\mathrm{P}$ in the cytoplasm matrix is low, it restricts the transport of triose phosphate from the chloroplasts which leads to accumulation of starch in the chloroplasts. 
This can be demonstrated by huge accumulation of starch granules in the chloroplast ultrastructure during drought stress (Fig. 2F). Fischer and Bremer (1993) believed that this phenomenon may be caused by the obstruction in the transport of the photoassimilates to the sink or the decrease in the utilization of photoassimilates. As an important micronutrient, Mo plays an essential role in electron donors/or acceptors in molybdoenzymes in plants, such as nitrogenase, aldehyde oxidase, xanthine dehydrogenase, and nitrate reductase catalyses (Kaufholdt et al. 2013). The reduction of Mo would hinder the metabolic processes of nitrogen, carbon, and sulfur in DS-treated plants.

When plants were exposed to low water potential, the ABA content increased rapidly in our study (Fig. $5 A$ ). ABA is considered to be an important messenger involved in the adaptive response of plants against abiotic and biotic stresses (Umezawa et al. 2006). ABA and IAA can improve plant drought-stress tolerance (Sreenivasulu et al. 2012, Defez et al. 2017). In this study, there was a slight increase but no significant difference in IAA compared with the WW group during drought stress, which may be attributed to the complexity of IAA biosynthesis regulation or/and the uniqueness of rice LYPJ. Many studies have shown that ABA can induce the expression of antioxidant enzymes to remove ROS (Jing and Zhang 2002, Shi et al. 2012). It was considered that the increased ABA would usefully act as a regulator in biotic responses in rice LYPJ. Additionally, it is known that ABA activates guard cell anion channels both by calcium-dependent and calcium-independent pathways; strong relationship between ABA and $\mathrm{Ca}^{2+}$ / calmodulin $(\mathrm{CaM})$ signaling system has been reported in the literature (Geiger et al. 2010). We speculated that $\mathrm{ABA}$ and $\mathrm{Ca}^{2+}$ form a highly ordered regulatory network in the regulation of plant responses to drought stress in rice LYPJ.

Taken together, evidence from physiological parameters and thylakoid ultrastructure analysis denoted that the changes induced by natural drought played a wider negative role in growth and photosynthetic processes in super high-yield hybrid rice during flowering stage and milk stage under drought stress. The suitable strategy is demonstrated by the positive correlation between changing their membrane lipid fluidity, variations of endogenous phytohormones, enhancement of osmotic substances, and stabilizing the grana thylakoids in order to minimize photoinhibition damage and ROS production in this super high-yield hybrid rice.

\section{References}

Abid M., Ali S., Qi L.K. et al.: Physiological and biochemical changes during drought and recovery periods at tillering and jointing stages in wheat (Triticum aestivum L.). - Sci. Rep.UK 8: 4615, 2018.

Arnon D.I.: Copper enzymes in isolated chloroplasts. Polyphenol oxidase in Beta vulgaris. - Plant Physiol. 24: 1-15, 1949.

Atkinson C.J.: The flux and distribution of xylem sap calcium to adaxial and abaxial epidermal tissue in relation to stomatal behaviour. - J. Exp. Bot. 42: 987-993, 1991.

Avila-Ospina L., Moison M., Yoshimoto K., Masclaux-Daubresse C.: Autophagy, plant senescence, and nutrient recycling. -
J. Exp. Bot. 65: 3799-3811, 2014.

Babu R.C., Shashidhar H.E., Lilley J.M. et al.: Variation in root penetration ability, osmotic adjustment and dehydration tolerance among accessions of rice adapted to rainfed lowland and upland ecosystems. - Plant Breeding 120: 233-238, 2008.

Blatt M.R., Gradmann D.: $\mathrm{K}^{+}$-sensitive gating of the $\mathrm{K}^{+}$outward rectifier in Vicia guard cells. - J. Membrane Biol. 158: 241-256, 1997.

Chen G.X., Liu S.H., Zhang C.J., Lu C.G.: Effects of drought on photosynthetic characteristics of flag leaves of a newlydeveloped super-high-yield rice hybrid. - Photosynthetica 42: 573-578, 2004.

Cheng S., Zhuang J., Fan Y.J. et al.: Progress in research and development on hybrid rice: A super-domesticate in China. Ann. Bot.-London 100: 959-966, 2007.

Cunningham F.X., Gantt E.: Genes and enzymes of carotenoid biosynthesis in plants. - Annu. Rev. Plant Phys. 49: 557-583, 1998.

Defez R., Andreozzi A., Dickinson M. et al.: Improved drought stress response in alfalfa plants nodulated by an IAA overproducing Rhizobium strain. - Front. Microbiol. 8: 2466, 2017.

Farooq M., Wahid A., Kobayashi N. et al.: Plant drought stress: effects, mechanisms and management. - Agron. Sustain. Dev. 29: 185-212, 2009.

Fischer E.S., Bremer E.: Magnesium deficiency in expanding leaves of Phaseolus vulgaris-gas exchange and nutrient concentrations. - Plant Soil 155: 419-422, 1993.

Geiger D., Scherzer S., Mumm P. et al.: Guard cell anion channel SLAC1 is regulated by CDPK protein kinases with distinct $\mathrm{Ca}^{2+}$ affinities. - P. Natl. Acad. Sci. USA 107: 8023-8028, 2010.

Gondor O.K., Szalai G., Kovács V. et al.: Impact of UV-B on drought- or cadmium-induced changes in the fatty acid composition of membrane lipid fractions in wheat. - Ecotox. Environ. Safe. 108: 129-134, 2014.

Guo Y.Y., Yu H.Y., Yang M.M. et al.: Effect of drought stress on lipid peroxidation, osmotic adjustment and antioxidant enzyme activity of leaves and roots of Lycium ruthenicum Murr. seedling. - Russ. J. Plant Physl+ 65: 244-250, 2018.

Hirschi K.D.: The calcium conundrum. Both versatile nutrient and specific signal. - Plant Physiol. 136: 2438-2442, 2004.

Hu W.H., Yan X.H., Xiao Y.A. et al.: 24-epibrassinosteroid alleviate drought-induced inhibition of photosynthesis in Capsicum annuum. - Sci. Hortic.-Amsterdam 150: 232-237, 2013.

Jing M.Y., Zhang J.H.: Water stress-induced abscisic acid accumulation triggers the increased generation of reactive and up-regulates the activities of antioxidant enzymes in maize leaves. - J. Exp. Bot. 53: 2401-2410, 2002.

Kaufholdt D., Gehl C., Geisler M. et al.: Visualization and quantification of protein interactions in the biosynthetic pathway of molybdenum cofactor in Arabidopsis thaliana. J. Exp. Bot. 64: 2005-2016, 2013.

Khanna-Chopra R.: Leaf senescence and abiotic stresses share reactive oxygen species-mediated chloroplast degradation. Protoplasma 249: 469-481, 2012.

Lanna A.C., Silva R.A., Ferraresi T.M. et al.: Physiological characterization of common bean (Phaseolus vulgaris L.) under abiotic stresses for breeding purposes. - Environ. Sci. Pollut. R. 25: 31149-31164, 2018.

Li L., Wang F., Yan P. et al.: A phosphoinositide-specific phospholipase $\mathrm{C}$ pathway elicits stress-induced $\mathrm{Ca}^{2+}$ signals and confers salt tolerance to rice. - New Phytol. 214: 1172-1187, 2017.

Lichtenthaler H.K.: Chlorophylls and carotenoids: Pigments 
of photosynthetic biomembranes. - Method. Enzymol. 148: 350-382, 1987.

Patakas A., Nikolaou N., Zioziou E. et al.: The role of organic solute and ion accumulation in osmotic adjustment in droughtstressed grapevines. - Plant Sci. 163: 361-367, 2002.

Shao R.X., Xin L.F., Zheng H.F. et al.: Changes in chloroplast ultrastructure in leaves of drought-stressed maize inbred lines. - Photosynthetica 54: 74-80, 2016.

Shi B., Ni L., Zhang A. et al.: OsDMI3 is a novel component of abscisic acid signaling in the induction of antioxidant defense in leaves of rice. - Mol. Plant 5: 1359-1374, 2012.

Shi J., Pan G.X.: [Effects of Cd-spiking treatment on Cd accumulation, subcellular distribution and content of nonprotein thiols in rice.] - Ecol. Env. Sci 24: 853-859, 2015. [In Chinese]

Sreenivasulu N., Harshavardhan V.T., Govind G. et al.: Contrapuntal role of ABA: Does it mediate stress tolerance or plant growth retardation under long-term drought stress? Gene 506: 265-273, 2012.

Sultana N., Ikeda T., Itoh R.: Effect of $\mathrm{NaCl}$ salinity on photosynthesis and dry matter accumulation in developing rice grains. - Environ. Exp. Bot. 42: 211-220, 1999.

Thomas P.G., Dominy P.J., Vigh L. et al.: Increased thermal stability of pigment protein complexes of pea thylakoids following catalytic hydrogenation of membrane lipids. BBA-Bioenergetics 849: 131-140, 1986.

Ullah A., Manghwar H., Shaban M. et al:: Phytohormones enhanced drought tolerance in plants: A coping strategy. Environ. Sci. Pollut. R. 25: 33103-33118, 2018.

Umezawa T., Fujita M., Fujita Y. et al.: Engineering drought tolerance in plants: discovering and tailoring genes to unlock the future. - Curr. Opin. Biotech. 17: 113-122, 2006.
Upchurch R.G.: Fatty acid unsaturation, mobilization, and regulation in the response of plants to stress. - Biotechnol. Lett. 30: 967-977, 2008.

Varone L., Ribas-Carbo M., Cardona C. et al.: Stomatal and non-stomatal limitations to photosynthesis in seedlings and saplings of Mediterranean species pre-conditioned and aged in nurseries: Different response to water stress. - Environ. Exp. Bot. 75: 235-247, 2012.

Vayssières A., Pěnčík A., Felten J. et al.: Development of the poplar-Laccaria bicolor ectomycorrhiza modifies root auxin metabolism, signaling, and response. - Plant Physiol. 169: 890-902, 2015.

Wang G.P., Li F., Zhang J. et al.: Overaccumulation of glycine betaine enhances tolerance of the photosynthetic apparatus to drought and heat stress in wheat. - Photosynthetica 48: 30-41, 2010.

Yamane K., Hayakawa K., Kawasaki M. et al.: Bundle sheath chloroplasts of rice are more sensitive to drought stress than mesophyll chloroplasts. - J. Plant Physiol. 160: 1319-1327, 2003.

Yang J., Zhang J.: Grain filling of cereals under soil drying. New Phytol. 169: 223-236, 2006.

Zhang M.P., Zhang C.J., Yu G.H. et al.: Changes in chloroplast ultrastructure, fatty acid components of thylakoid membrane and chlorophyll $a$ fluorescence transient in flag leaves of a super-high-yield hybrid rice and its parents during the reproductive stage. - J. Plant Physiol. 167: 277-285, 2010.

Zhou J.Y., Li X., Zhao D. et al.: Reactive oxygen species and hormone signaling cascades in endophytic bacterium induced essential oil accumulation in Atractylodes lancea. Planta 244: 699-712, 2016.

(C) The authors. This is an open access article distributed under the terms of the Creative Commons BY-NC-ND Licence. 